\title{
Rare Fungal Keratitis Caused by Coprinellus Radians
}

\author{
Xiuhai Lu $\cdot$ Xin Wang $\cdot$ Li Zhang $\cdot$ Xiaofeng Li $\cdot$ Xiaolin Qi
}

Received: 23 May 2019/Accepted: 4 December 2019/Published online: 8 January 2020

(C) The Author(s) 2020

\begin{abstract}
A case of fungal keratitis due to Coprinellus radians is reported. To our knowledge, fungal keratitis caused by this species was rare. Fungal hyphae were detected in corneal scrapings, and isolates were identified by morphology and by sequencing the internal transcribed spacer region of ribosomal DNA. The patient was treated with systemic and local antifungal therapy for 5 days, and lamellar keratoplasty was performed after no obvious improvement in symptoms. The in vitro antifungal susceptibilities of the case strain were tested for six antifungal agents. The results showed that 5-fluorouracil was resistant, fluconazole was moderately sensitive, and the other drugs assayed (amphotericin B, posaconazole, itraconazole and voriconazole) were highly effective against this fungus.
\end{abstract}

Keywords Fungal keratitis - Coprinellus radians . In vitro antifungal susceptibility test

Handling Editor: Philip Aloysius Thomas.

Xiuhai Lu and Xin Wang have contributed equally to this work.

X. Lu $\cdot$ X. Wang $\cdot$ L. Zhang $\cdot$ X. Li $\cdot$ X. Qi $(\bowtie)$

Shandong Eye Hospital, Shandong Eye Institute,

Shandong Academy of Medical Sciences, 372 Jingsi

Road, Jinan 250000, China

e-mail: qinglianqx1@163.com

\section{Introduction}

At present, more than 100 species that belong to 50 genera of fungi pathogens have been reported which cause human corneal infection [1]. The pathogens of fungal keratitis vary in different climatic regions. Filamentous fungi are the main pathogens throughout the world, especially in tropical and subtropical areas, and the most common pathogens are Fusarium and Aspergillus [1-5]. In China, the most common pathogenic agents are Fusarium, Aspergillus and Alternaria [6]. As a basidio-mycotic fungus, $C$. radians which is commonly known as a contaminant has not been reported as the cause of this infection. Therefore, we describe a case of keratitis due to $C$. radians.

\section{Case Report}

A 48-year-old female farm worker, her left eye was scratched by corn leaves 40 days ago, complained of foreign body sensation, with vision loss, lacrimation, photophobia and no other obvious discomfort. She was diagnosed with a corneal ulcer at local hospital and was given topical antibiotic eyedrops (specific medication is unknown), significant improvement in symptoms of self-discomfort. Two weeks later, her condition deteriorated, the symptoms such as eye abrasion and vision loss were significantly aggravated, accompanied by difficulty in opening eyes, so she 
went to the local hospital again and received systemic and topical anti-infection treatment, but because of no effect, she was referred to our hospital.

On first examination, the patient's vital signs were normal and her existing spectacle correction visual acuity was FC/20 cm in the left eye, which showed diffuse conjunctival hyperemia. There was a $5 \mathrm{~mm} \times \mathrm{mm}$ gray-white infiltration with irregular borders and edema in the center cornea, invasion of shallow and middle stroma (Fig. 1a). No abnormality was observed in the right eye.

On image logical examinations, anterior segment coherence optical tomography (AS-OCT) (Optovue, USA) showed that the central cornea infiltration depth was about $100 \mu \mathrm{m}$ and in vivo confocal microscopy (IVCM) (Heidelberg HRT3, RCM) showed that the accumulation of medium amount of necrotic material and the invasion depth of hyphae structure reached $200 \mu \mathrm{m}$, with a large number of chlamydospore-like structures clustered (Fig. 2). The endothelial cell count was about $2269 / \mathrm{mm}^{2}$.

Corneal scraping was performed at the bottom and edge of corneal ulcer, and placed on a microscope slide which drip 10\% potassium hydroxide; it showed abundant thick hyaline septate and branched hyphae, with abundant chlamydospore in the middle or terminal of the hyphae (Fig. 3a), while other scrapings were directly inoculated into 5\% sheep blood agar and Sabouraud glucose agar (SDA), and incubated at $28^{\circ} \mathrm{C}$ and $35{ }^{\circ} \mathrm{C}$, respectively. After $2-3$ days, small white colonies, apparently of a single fungus species, appeared on all the culture media.

After hyphae were detected, the patient was given voriconazole $10 \mathrm{mg} / \mathrm{ml}$ eyedrops four times per hour,
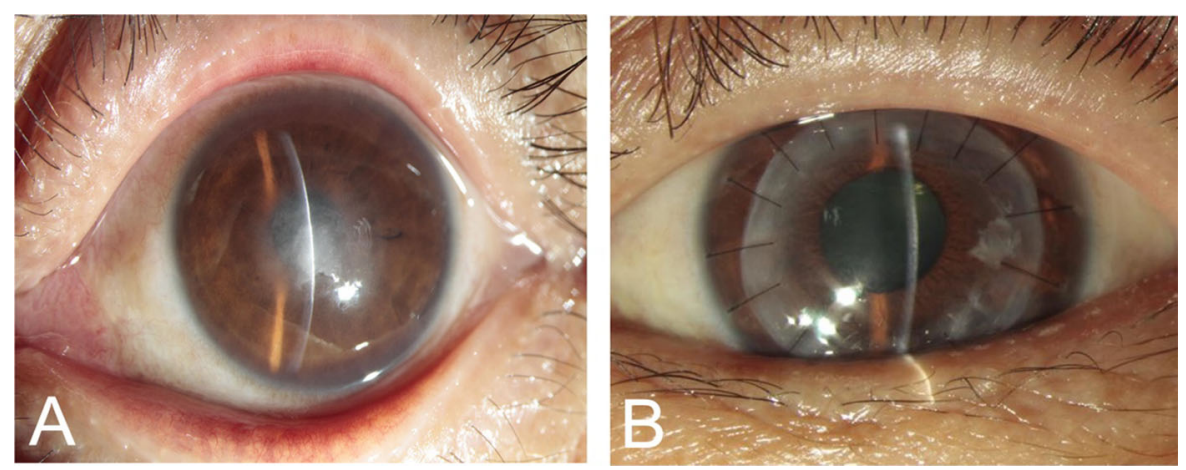

Fig. 1 a Slit-lamp examination of the left eye showing a $5 \times 5 \mathrm{~mm}^{2}$ gray-white lesion infiltrate at the center cornea, invasion of shallow and middle stroma, b lamellar keratoplasty after ineffective antifungal therapy
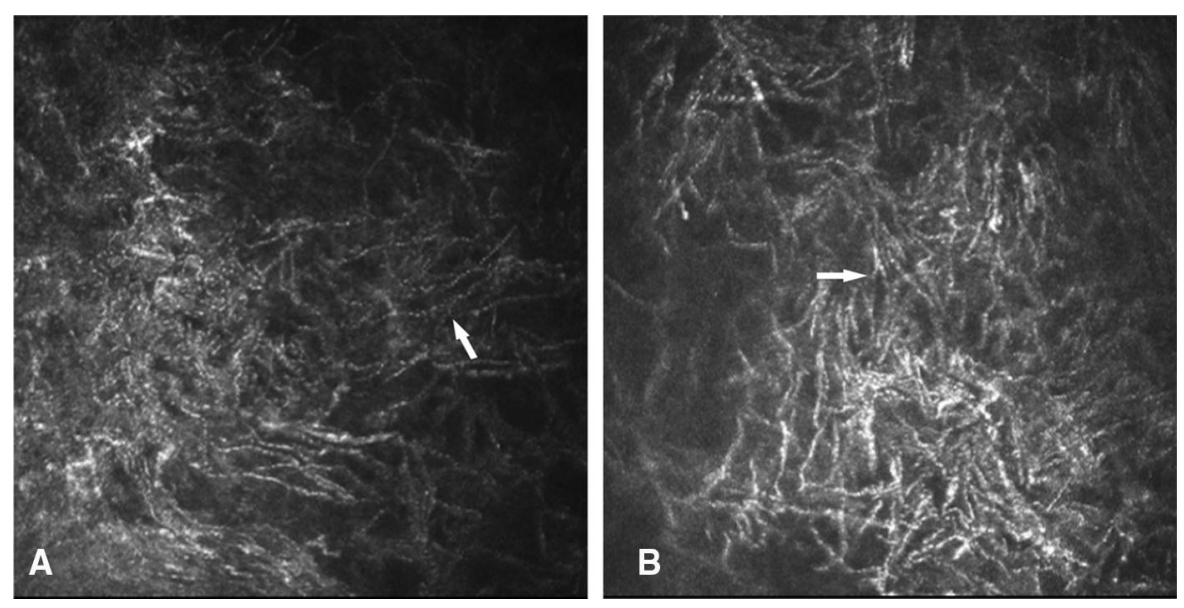

Fig. 2 Confocal microscopy, a a large number of chlamydospores are scattered in chains, $\mathbf{b}$ a large number of hyphae are densely distributed 


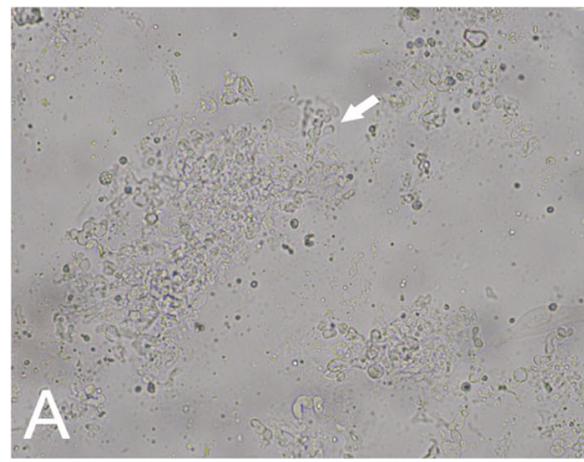

Fig. 3 a $10 \%$ potassium hydroxide smear showed a large number of complete hyphae, which were thick, septate, middle or terminal chlamydospore, solitary, chain-shaped or clustered-

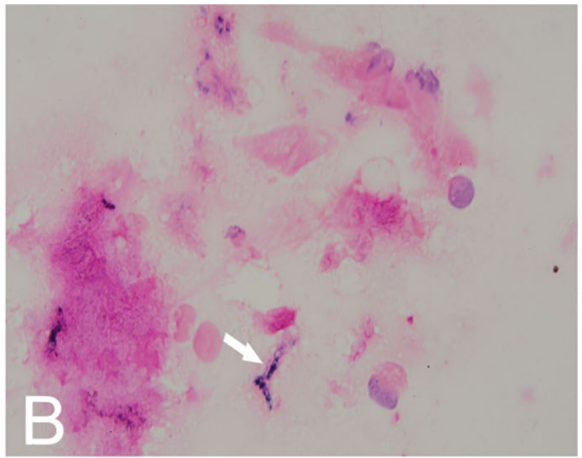

shaped (magnification of $\times 200$ ), b gram staining showed that the hyphae were wide and irregular in width (magnification of $\times 1000$ )
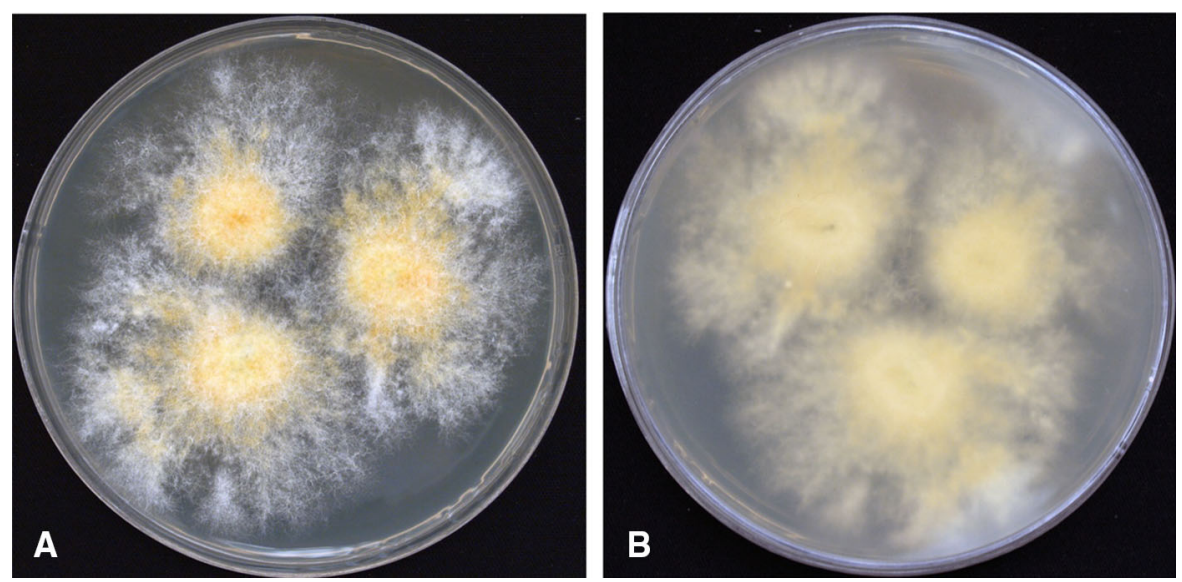

Fig. 4 a Colony morphology of SDA medium cultured at $28^{\circ} \mathrm{C}$ for 8 days showed that the colony was fluffy cotton flocculent and dark yellow in the middle, light yellow around, and $\mathbf{b}$ the reverse was yellow and yellow-brown. (Color figure online)

amphotericin B $0.25 \%$ eyedrops once an hour, levofloxacin $0.5 \%$ eyedrops four times a day, pranoprofen $0.1 \%$ eyedrops four times a day and ofloxacin $0.3 \%$ eye ointment once a night. Five days later, the inflammation did not subside. In order to avoid missing the operation opportunity, partial lamellar keratoplasty (glycerin preserved corneal tissue) was performed under peribulbar anesthesia after the patient signed the informed consent. After surgery, there was therapy with voriconazole $10 \mathrm{mg} /$ $\mathrm{ml}$ eyedrops once an hour, amphotericin B $0.25 \%$ eyedrops every two hours, ofloxacin $0.3 \%$ eye ointment four times a day and recombinant bovine basic fibroblast growth factor eye gel four times a day. Five days later, the voriconazole and amphotericin B $0.25 \%$ eyedrops were decreased to four times daily; at the same time, levofloxacin and pranoprofen eye drops were given four times a day. Eight days after the surgery, the corneal ulcer healed and the patient was discharged from hospital (Fig. 1b), and her visual acuity with spectacle correction improved to 20/200. The topical antifungals were tapered and discontinued, and outpatient follow-up was conducted.

\section{Mycology}

We identified fungi by morphology and molecular methods. The clinical isolate was subcultured on SDA and incubated in $28^{\circ} \mathrm{C}$. After 8 days, the colonies on SDA were floccose, with dark yellow fluffy aerial mycelia in the middle, light yellow around and the 


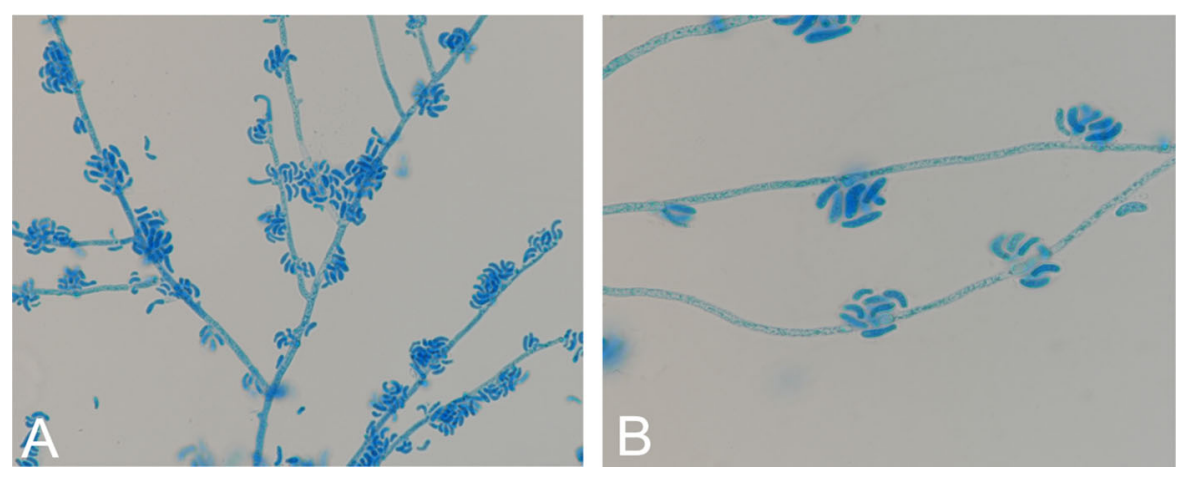

Fig. 5 SDA medium was cultured at 28 for 8 days and stained with lactophenol cotton blue staining (a magnification of $\times 400$, b magnification of $\times 1000$ )

Table 1 A checklist for submission of case report to Mycopathologia

\begin{tabular}{|c|c|c|}
\hline & Yes & No \\
\hline Original observation & $\square$ & $\square$ \\
\hline Ethical statement included & $\nabla$ & $\square$ \\
\hline Patient demographic anonymized & $\nabla$ & $\square$ \\
\hline $\begin{array}{l}\text { Title includes the term for medical and veterinary condition being described and the correct name of the } \\
\text { fungal pathogen (italicized) }\end{array}$ & $\nabla$ & $\square$ \\
\hline Title and abstract do not include superlatives such as 'new', 'first', 'unique', etc. & $\nabla$ & $\square$ \\
\hline Patient treatment and follow-up included & $\nabla$ & $\square$ \\
\hline Confirmatory, probable or possible diagnostic criteria defined Mycology & $\square$ & $\square$ \\
\hline Any author a specialist in fungal diagnostics? & $\square$ & $\square$ \\
\hline Culture positive with characteristic microscopy & $\nabla$ & $\square$ \\
\hline Culture confirmed with molecular tests & $\nabla$ & $\square$ \\
\hline Fungus name conform to current nomenclature & $\square$ & $\square$ \\
\hline Nucleotide sequence deposited in a known database & $\nabla$ & $\square$ \\
\hline Antifungal susceptibility test results included & $\nabla$ & $\square$ \\
\hline $\begin{array}{l}\text { Antifungal test result interpretation provided with reference to the guidance documents (CLSI, EUCAST) } \\
\text { Histopathology }\end{array}$ & $\nabla$ & $\square$ \\
\hline Any author a specialist in histopathology? & $\nabla$ & $\square$ \\
\hline High-resolution images included & $\nabla$ & $\square$ \\
\hline Specialized fungal stains used & $\nabla$ & $\square$ \\
\hline Differential histopathology diagnosis discussed & $\nabla$ & $\square$ \\
\hline
\end{tabular}


Table 2 Mycopathologia IMAGES checklist

\begin{tabular}{|c|c|c|}
\hline Original, unpublished observation being reported with ethical statement & $\square$ & $\square$ \\
\hline Report of potential interest to a mixed audience of physicians and laboratory scientists & $\square$ & $\square$ \\
\hline Brief title (three-four keywords) & $\nabla$ & $\square$ \\
\hline One paragraph description not to exceed 500 words & $\nabla$ & $\square$ \\
\hline No abstract, literature citation, bibliography or hyperlinks & $\square$ & $\square$ \\
\hline Maximum of four images with short legends (colored required for free online publication) & $\square$ & $\nabla$ \\
\hline $\begin{array}{l}\text { Author and primary affiliation at the end of the paragraph (name, degree, primary institution name, city, } \\
\text { state, country) }\end{array}$ & $\square$ & $\square$ \\
\hline
\end{tabular}

reverse was yellow and yellow-brown (Fig. 4). Microscopically, the hyphae were transparent, septated and branched. Conidiophore on both sides and tip of hyphae were short and thick. The conidia were single-celled, the ends were blunt, sausage-like, $\mathrm{S}$-shaped and C-shaped and clustered in slimy head (Fig. 5).

According to its morphological and microbial characteristics, it was preliminarily identified as Coprinellus spp.; however, the genus could not be ascribed to which species. To confirm the identification, the internal transcribed spacer (ITS) region was sequenced and DNA sequences were determined at Kingdom clinical trial center (Guangzhou, China). The resulting sequences were deposited in the GenBank database. Species identification was performed by searching databases using the BLAST sequence analysis tool (https://www.ncbi.nlm.nih. gov/BLAST/). The ITS sequence of the isolate was $99.69 \%$ similar to that of strain C. Radians.

\section{Antifungal Susceptibility Testing}

In vitro antifungal susceptibility testing was performed by Sensititre Yeast One ${ }^{\mathrm{TM}}$ (AccuMed International, Chicago, IL, USA) [7-10]. The isolate was subcultured on PDA plates and incubated at $28{ }^{\circ} \mathrm{C}$ for 7 days. The plates were covered with $0.85 \%$ saline, followed by gentle pipetting of the colonies with the tip of a sterile pipette, and adjustment of the concentration of suspension to 0.5 Maxwell turbidity. The incubator temperature was $28^{\circ} \mathrm{C}$, and reading results after $48 \mathrm{~h}$. The minimal inhibitory concentrations (MICs) for fluconazole, itraconazole, voriconazole, posaconazole, 5-fluorocytosine and amphotericin B were $16,0.25,0.015,0.25,>64$ and $0.25 \mu \mathrm{g} / \mathrm{ml}$, respectively (Table 1 ).

\section{Discussion}

Coprinellus radians belongs to large mushrooms in nature, subordinate to Basidiomycota, Agaricomycetes, Agaricomycetidae, Agaricales, Psathyrellaceae and Coprinellus.

Although the relevant information of $C$. radians was available in the previous literature, most of them were isolated from sputum specimens, diabetic feet, abdominal drainage fluid and skin ulcer wounds, which could not exclude the possibility of contamination, and there were few clinical reports of eye infection caused by this genus. The patient's left eye was scratched by corn leaf and had a long course of disease. Slit-lamp examination showed a gray-white ulcer lesion of about $5 \mathrm{~mm} \times 5 \mathrm{~mm}$ in the cornea central pupil area. Combining with corneal scrape histology, IVCM and culture results, it conformed to the predisposing causes, clinical manifestations and etiological diagnosis of ocular fungal infection.

Coprinellus radians has low nutritional requirements and can grow on a variety of media, the most suitable medium is SDA and PDA, and the culture time is about 10 days. The colony characteristics were obvious and grew rapidly on SDA medium. Its color is bright and like cotton flocculent, the middle is fluffy, 
dark yellow, and the surrounding is light yellow as well as yellow or yellow-brown on the back. The color gradually disappeared after many generations. The spores are single-celled, the ends are blunt, sausagelike, S-shaped and C-shaped and clustered in slimy head. Although the colonies and spores of the genus are distinctive and easy to identify, they should be distinguished from those of some Fusarium species in order to avoid confusion (Table 2).

In vitro drug sensitivity test showed that the MIC values of amphotericin $\mathrm{B}$, voriconazole, posaconazole and itraconazole were lower, while the MIC values of fluconazole and 5-fluorocytosine were higher. Due to the lack of a unified drug sensitivity break point, it is not yet possible to determine whether drug resistance exists. However, according to the MIC values, the rare genus was highly sensitive to amphotericin B, itraconazole, posaconazole and voriconazole, and resistant to fluconazole. Whether there is intrinsic drug resistance has not been reported. The patient was first treated in a primary hospitals and its specific medication was not clear; when she came to our hospital, the lesion had invaded the stroma, and sensitive antifungal agents and timely lamellar keratoplasty are effective. Therefore, fungal keratitis needs early diagnosis to identify different fungi genera and symptomatic treatment.

In summary, to our knowledge, this case of mycotic keratitis represents the first known reported infection caused by $C$. Radians, as well as being the first known reported in humans caused by this rare species.

We provided the checklist according to the request of the magazine [11].

Funding Funding was provided by the National Natural Science Foundation of China, (Grant Nos.: 81370989, 81570821), and the Young and Middle-Aged Scientists Research Awards Fung of Shandong Province (ZR2017BH004).

\section{Compliance with Ethical Standards}

Conflict of interest The authors declare that they have no conflict of interest and no ethical issue.

Open Access This article is licensed under a Creative Commons Attribution 4.0 International License, which permits use, sharing, adaptation, distribution and reproduction in any medium or format, as long as you give appropriate credit to the original author(s) and the source, provide a link to the Creative Commons licence, and indicate if changes were made. The images or other third party material in this article are included in the article's Creative Commons licence, unless indicated otherwise in a credit line to the material. If material is not included in the article's Creative Commons licence and your intended use is not permitted by statutory regulation or exceeds the permitted use, you will need to obtain permission directly from the copyright holder. To view a copy of this licence, visit http://creativecommons.org/licenses/by/4.0/.

\section{References}

1. Xie L, Zhai H, Zhao J, Sun S, Shi W, Dong X. Antifungal susceptibility for common pathogens of fungal keratitis in Shandong Province. China. Am J Ophthalmol. 2008;146(2):260-5.

2. Thomas Philip A. Current perspectives on ophthalmic mycoses. Clin Microbiol Rev. 2003;16(4):730.

3. Manikandan P, Abdel-Hadi A, Randhir Babu Singh Y, Revathi R, Anita R, Banawas S, et al. Fungal keratitis: epidemiology, rapid detection, and antifungal susceptibilities of and isolates from corneal scrapings. BioMed Res Int. 2019;2019(1):6395840.

4. Maharana PK, Farid M, Gelman R, Sinha R, Agarwal T, Titiyal JS, et al. Pharmacologic therapy of mycotic keratitis. Surv Ophthalmol. 2019;2(22):380-400.

5. Mahmoudi S, Masoomi A, Ahmadikia K, Tabatabaei SA, Soleimani M, Rezaie S, et al. Fungal keratitis: an overview of clinical and laboratory aspects. J Mycoses. 2018;61(12):916-30.

6. Zhong W-X, Xie L-X, Shi W-Y, Sun S-Y. Spectrum of infection of fungal keratitis:analysis of 654 cases. Natl Med J China. 2006;86(24):1681-5.

7. Pujol I, Guarro J, Llop C, Soler L, Fernandez-Ballart J. Comparison study of broth macrodilution and microdilution antifungal susceptibility tests for the filamentous fungi. Antimicrob Agents Chemother. 1996;40(9):2106-10.

8. Clinical, Laboratory Standards Institute. Reference method for Broth dilution antifungal susceptibility testing of filamentous fungi: approved standard-M38. 3rd ed. Wayne: CLSI; 2017.

9. Carrillo Munoz AJ, Quindos G, Ruesga M, Del Valle O, Peman J, Canton E, et al. In vitro antifungal susceptibility testing of filamentous fungi with Sensititre Yeast One. Mycoses. 2010;49(4):293-7.

10. Espinel-Ingroff A, Warnock DW, Vazquez JA, ArthingtonSkaggs BA. In vitro antifungal susceptibility methods and clinical implications of antifungal resistance. Med Mycol. 2000;38(s1):293-304.

11. Bouchara JP, Chaturvedi V. The curious case of "Case Report" of infections caused by human and animal fungal pathogens: an educational tool, an online archive, or a format in need of retooling. Mycopathologia. 2018;183(6):879-91. https://doi.org/10.1007/s11046-0180314-1.

Publisher's Note Springer Nature remains neutral with regard to jurisdictional claims in published maps and institutional affiliations. 